\title{
Genomic Epidemiology and Evolution of Duck Hepatitis A Virus
}

\author{
Enikő Fehér ${ }^{1}$, Szilvia Jakab ${ }^{1}$, Krisztina Bali ${ }^{1}$, Eszter Kaszab ${ }^{1}$, Borbála Nagy ${ }^{1}$, Katalin Ihász ${ }^{1}$, Ádám Bálint ${ }^{2}$, \\ Vilmos Palya ${ }^{3}$ (D) and Krisztián Bányai ${ }^{1,4, *}$
}

1 Veterinary Medical Research Institute, Hungária krt 21, H-1143 Budapest, Hungary; feher.eniko@vmri.hu (E.F.); jakab.szilvia@vmri.hu (S.J.); bali.krisztina@vmri.hu (K.B.); kaszab.eszter@vmri.hu (E.K.); nagy.borbala@vmri.hu (B.N.); ihasz.katalin@vmri.hu (K.I.)

2 Veterinary Diagnostic Directorate, National Food Chain Safety Office, Tábornok utca 2, H-1143 Budapest, Hungary; BalintAd@nebih.gov.hu

3 Ceva-Phylaxia Veterinary Biologicals Co., Ltd., H-1107 Budapest, Hungary; vilmos.palya@ceva.com

4 Department of Pharmacology and Toxicology, University of Veterinary Medicine, István utca 2, H-1078 Budapest, Hungary

* Correspondence: bkrota@hotmail.com

Citation: Fehér, E.; Jakab, S.; Bali, K.; Kaszab, E.; Nagy, B.; Ihász, K.; Bálint, Á.; Palya, V.; Bányai, K. Genomic Epidemiology and Evolution of Duck Hepatitis A Virus. Viruses 2021, 13, 1592. https://doi.org/10.3390/ v13081592

Academic Editor:

François-Loïc Cosset

Received: 17 June 2021

Accepted: 6 August 2021

Published: 11 August 2021

Publisher's Note: MDPI stays neutral with regard to jurisdictional claims in published maps and institutional affiliations.

Copyright: (c) 2021 by the authors. Licensee MDPI, Basel, Switzerland. This article is an open access article distributed under the terms and conditions of the Creative Commons Attribution (CC BY) license (https:// creativecommons.org/licenses/by/ $4.0 /)$.

\begin{abstract}
Duck hepatitis A virus (DHAV), an avian picornavirus, causes high-mortality acute disease in ducklings. Among the three serotypes, DHAV-1 is globally distributed, whereas DHAV-2 and DHAV-3 serotypes are chiefly restricted to Southeast Asia. In this study, we analyzed the genomic evolution of DHAV-1 strains using extant GenBank records and genomic sequences of 10 DHAV-1 strains originating from a large disease outbreak in 2004-2005, in Hungary. Recombination analysis revealed intragenotype recombination within DHAV-1 as well as intergenotype recombination events involving DHAV-1 and DHAV-3 strains. The intergenotype recombination occurred in the VP0 region. Diversifying selection seems to act at sites of certain genomic regions. Calculations estimated slightly lower rates of evolution of DHAV-1 (mean rates for individual protein coding regions, $5.6286 \times 10^{-4}$ to $1.1147 \times 10^{-3}$ substitutions per site per year) compared to other picornaviruses. The observed evolutionary mechanisms indicate that whole-genome-based analysis of DHAV strains is needed to better understand the emergence of novel strains and their geographical dispersal.
\end{abstract}

Keywords: duck hepatitis A virus; recombination; Hungary

\section{Introduction}

Infection caused by duck hepatitis virus 1 (DHV-1 or DHV type I), recently renamed as duck hepatitis A virus (DHAV), may be fatal in up to $95 \%$ of ducklings, typically under three weeks of age [1-3]. The disease is characterized by hepatic failures (enlargement, hemorrhages and necrosis), neurological signs (ataxia, opisthotonus) and the sudden death of affected birds [1-4]. The duck industry has been heavily affected by this virus; however, the disease can be efficiently prevented by vaccination [5].

DHAV belongs to the Avihepatovirus genus of the Picornaviridae family (https:/ / talk. ictvonline.org/taxonomy / accessed on 7 August 2021). The viral genome is a 7.7-kilobaselong positive-sense single stranded RNA and consists of a single large open reading frame (ORF) flanked by the $5^{\prime}$ and $3^{\prime}$ non-coding regions. The polyprotein encoded by the single ORF is predicted to code for the VP0, VP3, and VP1 structural proteins and the 2A1, 2A2, 2B, 2C, 3A, 3B, 3C, and 3D non-structural proteins [6,7].

Three serotypes/genotypes (DHAV-1, DHAV-2 and DHAV-3) of DHAV have been distinguished by serological and phylogenetic analyses. Outbreaks of DHAV-1 have been reported in many parts of the world, whereas DHAV-2 and DHAV-3 seemed to be geographically restricted to East and South Asia, although a DHAV-3 outbreak has recently been reported in Egypt (Figure 1) [1,2,6-18]. 


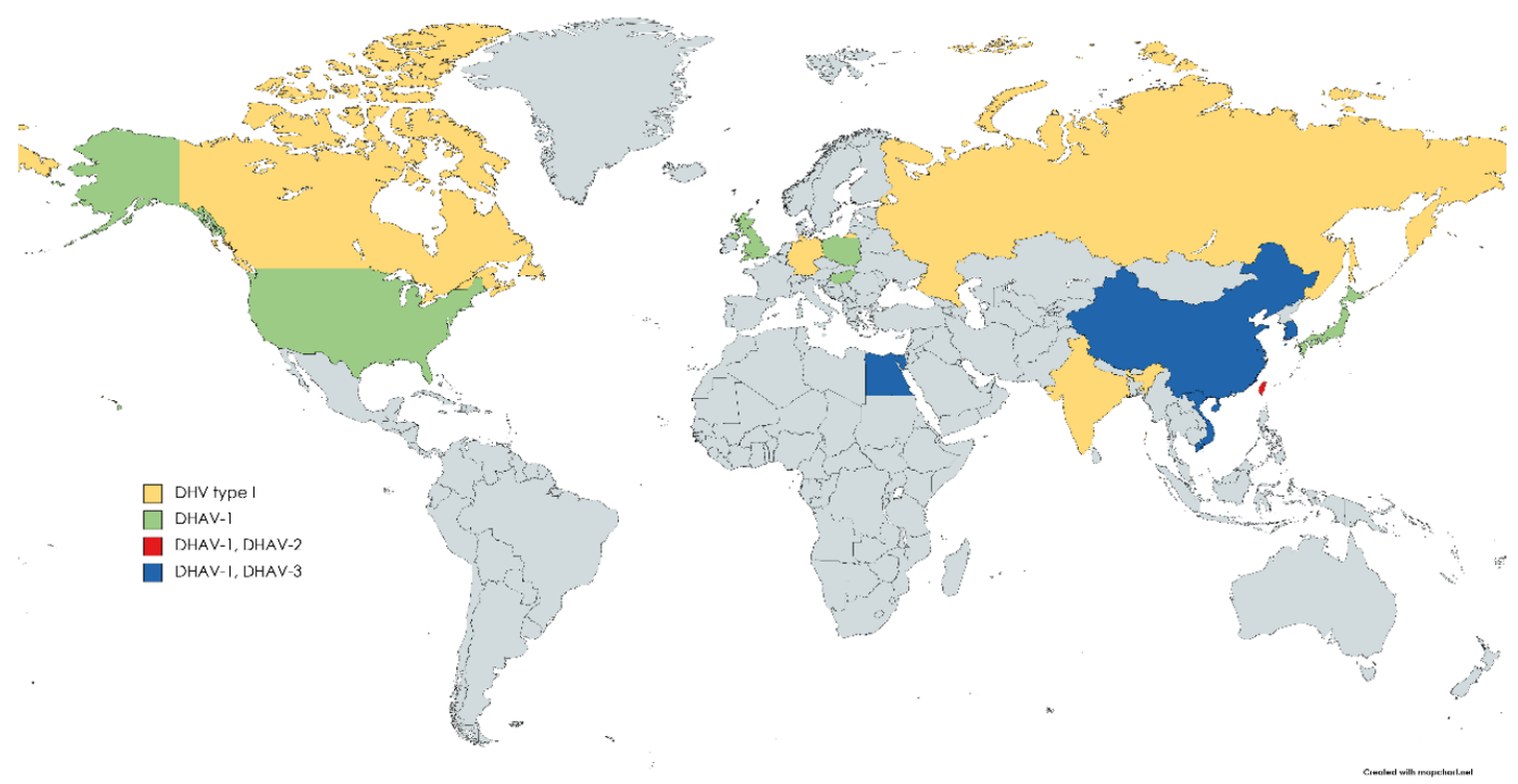

Figure 1. Geographic distribution of DHAV serotypes/genotypes. DHV type I is a historic assignment of DHAV introduced prior to the separation of serotypes.

Outbreaks due to DHAV were frequently reported in Hungary until the early 1990s. Thereafter, perhaps due to the adequate control and prevention measures, occurrence of the disease was recorded only once, during an epizootic in 2004-2005 that affected 112 flocks of Pekin ducks and Muscovy ducks [18]. In the present study, we collected DHAV sequences from GenBank and from the archival sample that contained isolates from the 2004-2005 Hungarian disease outbreak, and performed molecular evolutionary analyses.

\section{Materials and Methods}

\subsection{Viruses and Sequencing}

All 10 Hungarian isolates available for the study originated from different duck farms in Bács-Kiskun County, Hungary. The carcasses were sent for duck hepatitis virus testing to the Veterinary Diagnostic Directorate, National Food Chain Safety Office, Budapest, Hungary. Liver samples were collected at autopsy and processed for virus isolation in embryonated Muscovy duck eggs as well as for diagnostic PCR.

For genome sequencing, the viral RNA was extracted from homogenized liver of the embryos using the DirectZol MiniPrep kit (Zymo Research, Irvine, CA, USA). For complete genome amplification, the viral RNA was reverse-transcribed with SuperScript ${ }^{\mathrm{TM}}$ III Reverse Transcriptase (Thermo Fisher Scientific, Waltham, MA, USA) and specific reverse primers (Table 1). The mixture was incubated at $55^{\circ} \mathrm{C}$ for $60 \mathrm{~min}$ and at $70{ }^{\circ} \mathrm{C}$ for $15 \mathrm{~min}$. Three overlapping fragments of the genome were amplified in $25 \mu \mathrm{L} \mathrm{PCR} \mathrm{mixtures}$ containing Phusion DNA Polymerase according to the manufacturers' instructions and at $58{ }^{\circ} \mathrm{C}$ primer annealing temperature (Thermo Fisher Scientific, Waltham, MA, USA).

Table 1. Primer sequences used for the reverse transcription and amplification of duck hepatitis A virus genomes in this study.

\begin{tabular}{ccc}
\hline Primer Name & Orientation & $\mathbf{5}^{\prime}-\mathbf{3}^{\prime}$ Sequence \\
\hline DHAV_F & forward & GGAGGTGGTGCTGAAA \\
DHAV_R1 & reverse & GTGGAAAGAAGAGAGCTGGC \\
DHAV_F2 & forward & GATGTGGCATGTTGTYAAYCGAC \\
DHAV_R2 & reverse & CAACCAAAYGCTGATGTTTTGG \\
DHAV_F3 & forward & CCAACYCTTCARTGGCTCCAG \\
DHAV_R3 & reverse & CATTTGGCTTAGGGTCCTCAC \\
\hline
\end{tabular}


The PCR products were mixed for each isolate to obtain equimolar ratios for the three partially overlapping amplicons, and cDNA libraries were prepared for sequencing with an Ion Torrent PGM ${ }^{\mathrm{TM}}$ or an Illumina NextSeq ${ }^{\mathrm{TM}} 500$ platform. Ion Torrent-compatible DNA libraries were prepared using the NEBNext ${ }^{\circledR}$ Fast DNA Fragmentation \& Library Prep Set for Ion Torrent (New England Biolabs, Ipswich, MA, USA) with the Ion Xpress ${ }^{\text {TM }}$ Barcode Adapters (Thermo Fisher Scientific, Waltham, MA, USA) according to the protocol recommended by the manufacturers. The emulsion PCR and subsequent template enrichment were performed with the Ion PGM ${ }^{\mathrm{TM}}$ Template IA 500 Kit (Thermo Fisher Scientific, Waltham, MA, USA) and the Ion Torrent One Touch ES module, respectively. Sequencing was carried out on an Ion $316^{\mathrm{TM}}$ Chip (Thermo Fisher Scientific, Waltham, MA, USA) using the Ion Torrent PGM ${ }^{\mathrm{TM}}$. The cDNA libraries for Illumina sequencing were prepared by using the Nextera ${ }^{\circledR}$ XT DNA Library Preparation Kit (Illumina, San Diego, CA, USA) and the Nextera ${ }^{\circledR}$ XT Index Kit v2 Set A (Illumina, San Diego, CA, USA) according to the protocol of the manufacturer. The $\mathrm{i} 5$ and $\mathrm{i} 7$ index primers were incorporated into library DNA via 12 PCR cycles. Libraries were purified, quantified and then pooled; denatured library DNAs were sequenced using an Illumina ${ }^{\circledR}$ NextSeq $^{\mathrm{TM}} 500$ sequencer using the NextSeq $^{\mathrm{TM}}$ 500/550 Mid Output Kit (Illumina, San Diego, CA, USA).

GenBank sequence records were collected in November 2020 and checked for new entries in May 2021. In total, 114 DHAV-1 (58 complete genomes and 56 VP1 genes) and 24 DHAV-3 (all complete genomes) sequences with available information on the date and place of collection were retrieved from GenBank. The time of origin of downloaded reference sequences encompassed a period from 1993 to 2020; sequences originated from Asia (China, Taiwan, Korea, Vietnam) and Africa (Egypt). Sequence data from the Hungarian DHAV-1 strains were generated during 2020.

\subsection{Sequence Analysis}

Complete coding sequences from short-read sequences were assembled with mapping to references using the Geneious Prime ${ }^{\circledR}$ v.2020.2.4 package (16 September 2020; Biomatters, Auckland, New Zealand). The final consensus lengths of DHAV genomes were 7370-7386 nucleotides (95.6-96\% coverage compared with reference strains KF953535, FJ496341 and KP721458). Regarding the sequencing results generated with Ion Torrent PGM system, 11,615 to 89,998 reads were mapped to the reference sequences, whereas the corresponding data for Illumina sequencing were 643,365 to 947,046 reads.

The BLASTn engine was used to determine similarities of the genomic sequences with relevant sequences in GenBank. Sequence alignments were prepared using the Geneious Prime $^{\circledR}$ V.2020.2.4 (16 September 2020; Biomatters, Auckland, New Zealand) and AliView (9 March 2021, Uppsala University, Sweden) software [19]. Pairwise sequence identities were calculated using MEGA6 v6.06 (April 2014) with the $p$-distance and pairwise deletion options [20].

Recombination analysis was carried out using RDP4 v4.101 software using the automated suite of algorithms with default settings [21]. The highest acceptable $p$-value was set to 0.05 . Possible recombination signals were accepted if they were supported by independent methods with $p$-values of $\leq 10^{-6}$, after Bonferroni correction for multiple comparisons had been implemented in the program. The significant evidence of recombination, confirmed by phylogenetic analysis, was taken to represent strong evidence for recombination. The multiple-aligned sequences were cut into sections at the positions where the putative recombination breakpoints were identified, and phylogenetic analyses were carried out with MEGA6 v6.06 (April 2014) software, using the neighbor-joining method with a $p$-distance model and 1000 bootstrap replicates.

Selection constraints and the ratios of non-synonymous to synonymous mutations $(\mathrm{dN} / \mathrm{dS}$ ) were calculated for the coding sequences with the FUBAR (Fast Unconstrained Bayesian AppRoximation), FEL (Fixed Effects Likelihood) and MEME (Mixed Effects Model of Evolution) models of the Datamonkey server [22]. The positive selection results detected 
with FEL and MEME analyses were considered significant at $p=0.05$, whereas a posterior probability of $\geq 0.95$ was set for FUBAR calculations.

The temporal structure of the sequence dataset was checked with TempEst v1.5.3 (13 June 2019) software prior to evolutionary analysis, applying the Markov chain Monte Carlo method implemented in the BEAST package 1.10.4 (11 November 2018) [23-25]. After running multiple tests with various parameters, in the final analyses, the sequences were tested using lognormal relaxed molecular clock, constant size coalescent tree prior and 50 million iterations. The best fit substitution model was estimated with the smart model selection tool in PhyML 3.0 software [26]. The effective sample size and evolutionary rates were extracted with Tracer v1.7.1 [27]. The confidence level in each parameter estimate was given by the $95 \%$ highest posterior density (HPD) values. A maximum clade credibility tree was generated with TreeAnnotator v1.10.4 and visualized FigTree v1.4.2 (9 July 2014) [24,28].

\section{Results and Discussion}

When analyzing the newly determined near-complete genomes of Hungarian genotype DHAV-1 strains, the BLASTn engine found significant hits with Asian-origin DHAV-1 sequences with up to $99.85 \%$ identities (at $100 \%$ coverage). Short genomic fragments (derived from partial GenBank sequence records) were similar to Egyptian-origin DHAV-1 VP1 sequences with up to $99.4 \%$ identities.

Full-length and partial genome sequences of DHAV isolates were retrieved from GenBank for further analyses. First, we investigated whether recombination affected the DHAV genomes in our data-set. Although traces of intragenotype recombination have previously been described [9], the improved methodology and the increasing number of sequence information enabled more accurate analyses to be performed. Our analyses indicated that recombination events affected the viral genomes of DHAV- 1 and DHAV-3 strains, and some recombinants seemed to have acquired RNA fragments from parental strains belonging to different DHAV genotypes (Table 2, Figure 2).

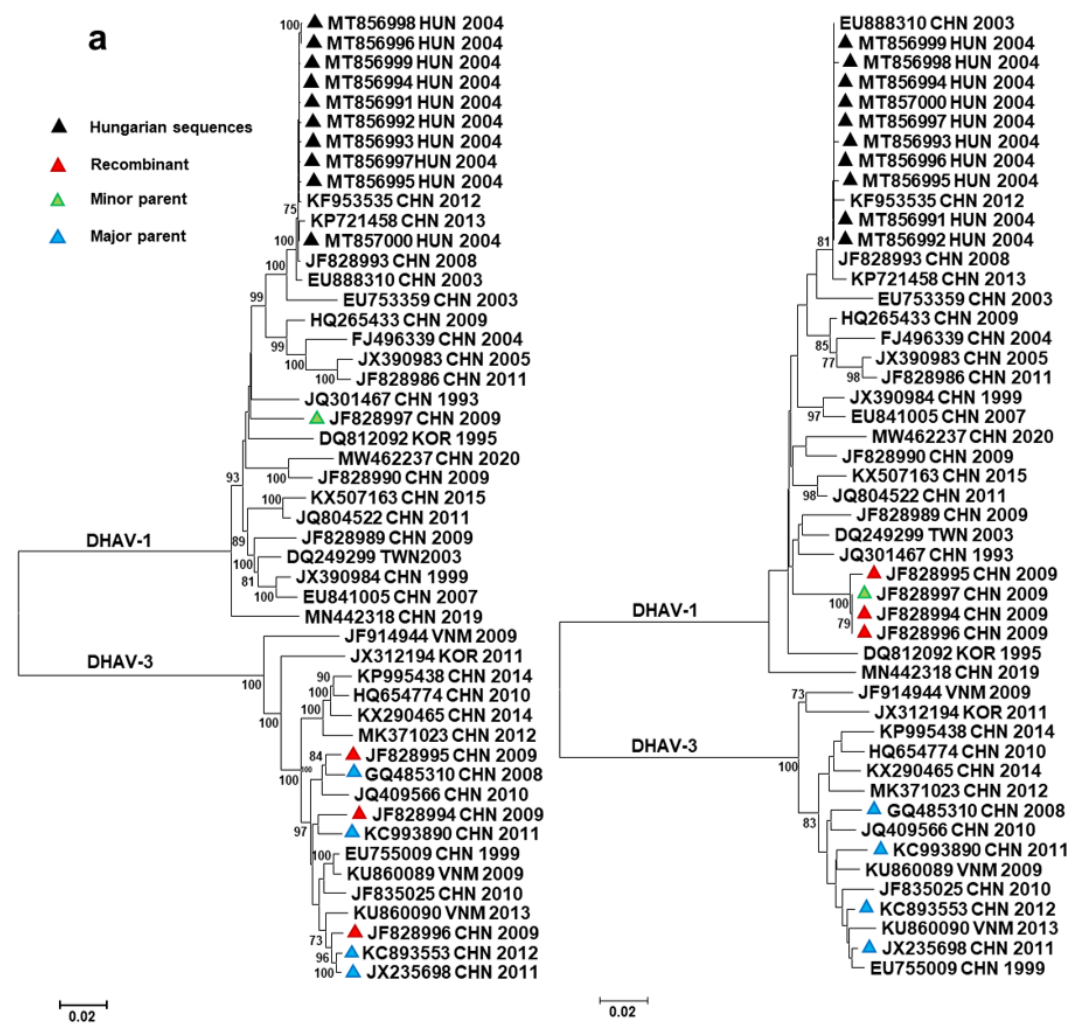

Figure 2. Cont. 


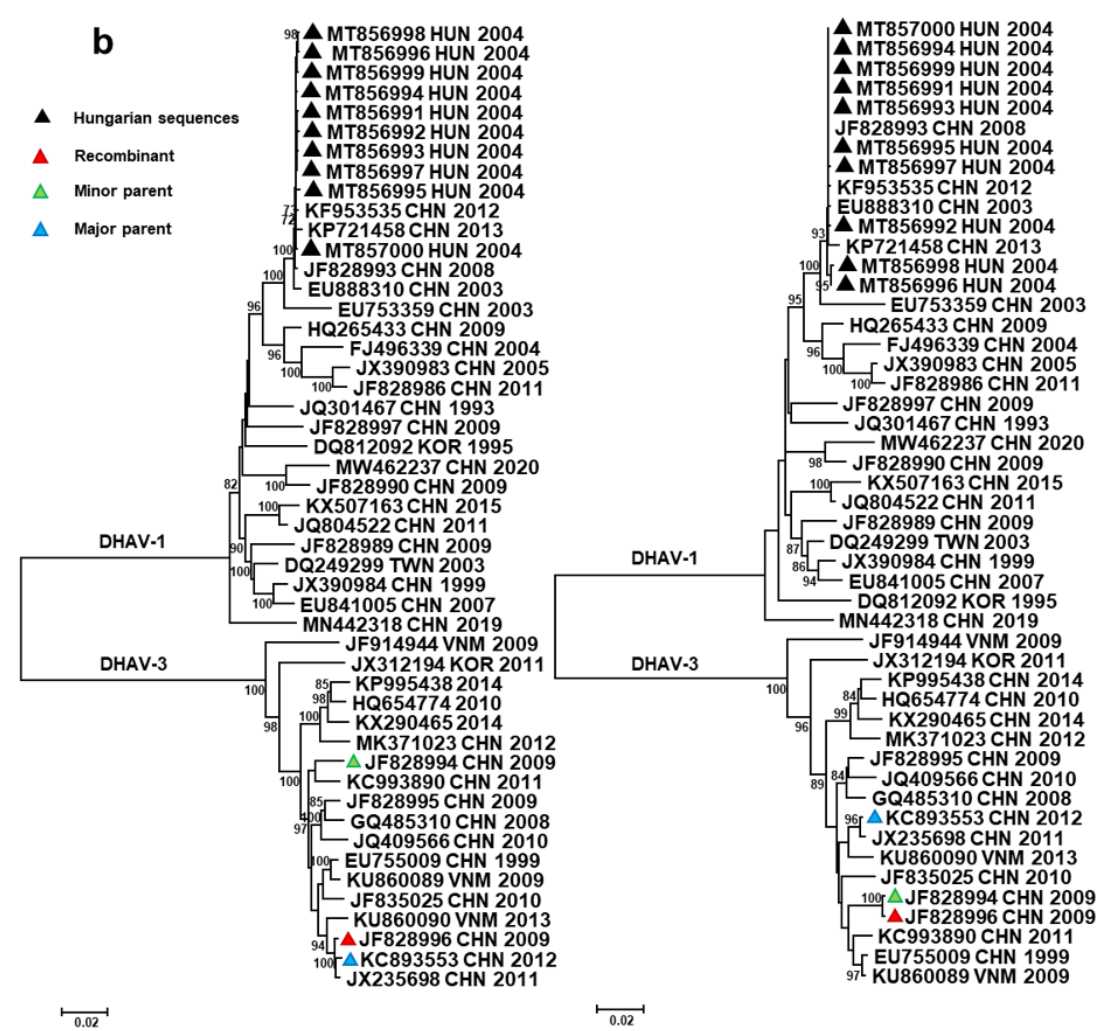

Figure 2. Unrooted neighbor-joining phylogenetic trees representing intergenotypic (a) and intragenotypic (b) recombination events. The trees were generated with a $p$-distance model (1000 bootstrap replicates) using sequence alignments extracted from polyprotein sequences according to the predicted recombination points determined with RDP4 software. The trees show groupings of recombinant strains with the putative major (tree on the left) and minor (tree on the right) parents (or related strains with GenBank records in all cases when true parents are unknown).

Table 2. Inter- and intragenotype recombination events affecting DHAV genomes, as revealed by recombination analysis.

\begin{tabular}{ccccccc}
\hline Event $^{*}$ & Region & Recombinant & Major Parent & Minor Parent & Recombination Type & $p$-Values \\
\hline$\# 1$ & VP0 & JF828994-996 ** & JX235698, KC993890, GQ485310 & JF828997 & Intergenotype & $10^{-11}-10^{-176}$ \\
$\# 2$ & 3A-3D & JF828996 & KC893553 & JF828994 & Intragenotype & $10^{-8}-10^{-16}$ \\
$\# 3$ & VP0 & EU888310 & MT856991 to MT857000 & DQ249299 & Intragenotype & $10^{-6}-10^{-7}$ \\
\hline
\end{tabular}

* Three recombination events were identified. These events were arbitrarily assigned. ${ }^{* *}$ Assignment of genotypes: normal letter, DHAV-1 sequence; boldface, DHAV-3 sequence.

In three Chinese DHAV-1/DHAV-3 intergenotype recombinants (Recombination event 1 in Table 2, Figure 2a), an $\sim 800 \mathrm{nt}$ long fragment of the $5^{\prime}$ genomic regions shared high sequence identity with DHAV-1 sequences (GenBank acc. no., JF828997), whereas the remaining $7 \mathrm{~kb}$ long genomic regions were aligned with DHAV-3 sequences (GenBank acc. no., JX235698, KC993890, GQ485310, KC893553). The recombination crossover involved the VP0 capsid-encoding region of the polyprotein in these strains. The viral protein VP0 has marked immunogenic features; thus, changes in the corresponding genomic region may contribute to immune escape mechanisms and also may influence vaccine development [5]. Of interest, although the sequence divergence within the DHAV-1 origin VP0 of Chinese intergenotype recombinants was negligible, the DHAV-3 origin genomic fragments showed marked variation, with $95.8 \%$ to $99.8 \%$ sequence identity with each other and their respective homologous sequences. This finding is consistent with the hypothesis that multiple independent DHAV-3 field strains acquired the VP0 region of a single circulating DHAV-1 parent. Of interest, an intragenotype recombination event (Recombination event 2 in Table 2, see also Figure $2 \mathrm{~b}$ ) affected a $\sim 1300 \mathrm{nt}$ long part of the 3A-3D region of the 
above-mentioned recombinant strain (GenBank acc. no., JF828996), in which case the major parent may have been a DHAV-3 strain very similar to a reference strain collected in China in 2012 (GenBank acc. no., KC893553), whereas the minor parent may have been another intergenotype recombinant strain (GenBank acc. no., JF828994). Strains very similar to the Hungarian strains (GenBank acc. no., MT856991 to MT857000) were also predicted to serve as putative major parents in a recombination event (Recombination event 3 in Table 2) donating a 190 nt long fragment of the VP0 coding region to a strain collected in China in 2003 (GenBank acc. no., EU888310); in this case, the putative minor parent was similar to a strain collected in Taiwan in 2003 (GenBank acc. no., DQ249299). Although only two methods of analysis supported this event, the sequence and phylogenetic analyses (data not shown) also suggested a historic recombination event.

Next, we omitted all putative recombinants whose inclusion could have affected subsequent analyses. In the investigation of Darwinian evolution, we focused on DHAV-1, where all Hungarian strains belonged. Selection constraints were calculated for the DHAV-1 coding sequences with various models. The overall $\mathrm{dN} / \mathrm{dS}$ ratio was low $(\leq 0.159)$ for the polyprotein region and single genes. Positive selection was predicted to act at some aa sites, primarily in the capsid-coding VP0, VP3 and VP1 regions (Figure 3). Applying strict statistical settings, four sites were highlighted in the coding region as potential sites under positive selection constraints, including aa542 (S49PLT in VP1), aa676 (Q183RGHY in VP1), aa680 (D187VG in VP1) and aa1719 (Q104HR in 3C) of the polyprotein (Figure 3). The aa sites 183 and 187 belong to the hypervariable region of the VP1; aa substitutions of this region were connected to changes in virulence. Furthermore, changes at other sites of the VP1, as well as the VP0 and VP3 regions, may affect the virulence or facilitate immune escape mechanisms [1,29-31].

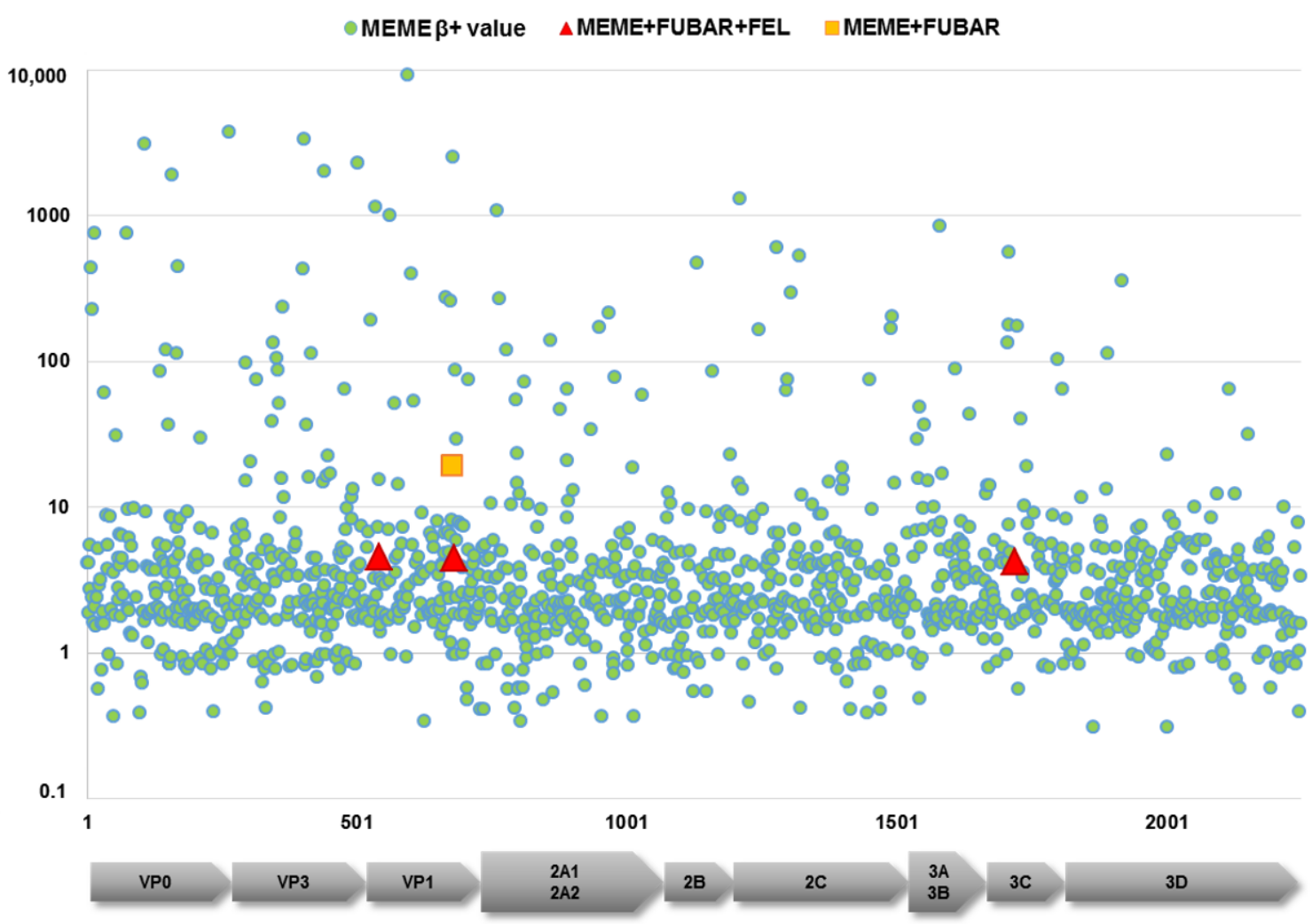

Figure 3. Results of the selection constraint analysis. $\beta+$ values ( $y$-axis) at individual sites ( $x$-axis) were extracted and plotted from results of the MEME analysis (green circles). Sites under positive selection predicted with high significance values are labeled with red triangles and the yellow square. 
Evolutionary rates were estimated for each DHAV-1 genomic region coding for a mature protein. The rates for single genes were in the range of $5.6286 \times 10^{-4}$ to $1.1147 \times 10^{-3}$ (Table 3); these values were slightly lower than those seen in a number of other viruses within the Picornavirales order [32,33]. In these analyses, the polymerase-encoding 3D region showed the lowest values. In a previous study, Ma et al. [30] estimated a substitution rate of $5.57 \times 10^{-4}\left(95 \%\right.$ HPD $2.66 \times 10^{-4}$ to $\left.8.45 \times 10^{-4}\right)$ for DHAV-1 VP1 sequences. Analysis of an extended sample set of DHAV-1 sequences (including complete and partial genome sequences), described from strains collected between 1993 and 2020 showed a mean value of $1.013 \times 10^{-3}\left(95 \%\right.$ HPD $6.7481 \times 10^{-4}$ to $\left.1.3458 \times 10^{-3}\right)$ for this genomic region, which was still lower than the average rates calculated for other picornaviruses $[32,33]$.

Table 3. The $\mathrm{dN} / \mathrm{dS}$ ratios and evolutionary rates (substitutions/site/year) estimated for individual genes of DHAV-1.

\begin{tabular}{ccccc}
\hline Region & Model & dN/dS & Evolutionary Rate s/s/y & 95\% HPD \\
\hline VP0 & HKY + G & 0.0945 & $7.0037 \times 10^{-4}$ & $2.3584 \times 10^{-4}$ to $1.2087 \times 10^{-3}$ \\
VP1 & HKY + G & 0.159 & $7.8508 \times 10^{-4}$ & $3.1147 \times 10^{-4}$ to $1.2961 \times 10^{-3}$ \\
VP3 & HKY + G & 0.105 & $9.0959 \times 10^{-4}$ & $3.6255 \times 10^{-4}$ to $1.5174 \times 10^{-3}$ \\
2A1 & HKY & 0.087 & $1.1147 \times 10^{-3}$ & $2.5081 \times 10^{-4}$ to $2.2143 \times 10^{-3}$ \\
2A2 & HKY + G & 0.111 & $8.3308 \times 10^{-4}$ & $3.0703 \times 10^{-4}$ to $1.3598 \times 10^{-3}$ \\
2B & HKY + G & 0.0489 & $8.112 \times 10^{-4}$ & $2.2148 \times 10^{-4}$ to $1.4842 \times 10^{-3}$ \\
2C & HKY + G & 0.0472 & $6.1681 \times 10^{-4}$ & $2.0083 \times 10^{-4}$ to $1.0726 \times 10^{-3}$ \\
3A & GTR + G & 0.0545 & $8.1151 \times 10^{-4}$ & $2.1100 \times 10^{-4}$ to $1.5012 \times 10^{-3}$ \\
3B & HKY + G & 0.127 & $1.059 \times 10^{-3}$ & $2.5708 \times 10^{-4}$ to $1.9493 \times 10^{-3}$ \\
3C & HKY + G & 0.0466 & $6.8276 \times 10^{-4}$ & $2.7878 \times 10^{-4}$ to $1.1399 \times 10^{-3}$ \\
3D & HKY + G & 0.0719 & $5.6286 \times 10^{-4}$ & $1.8270 \times 10^{-4}$ to $9.9198 \times 10^{-4}$ \\
VP1 extended sequence set & HKY + G + I & 0.191 & $1.013 \times 10^{-3}$ & $6.7481 \times 10^{-4}$ to $1.3458 \times 10^{-3}$ \\
\hline
\end{tabular}

In time-scaled phylogenetic analyses of DHAV-1 sequences, some bias in sample selection was seen, given that sequences were available for strains collected over a 24-year period (and only three sequences were from before 2000) and represented only three major geographic areas: Far East Asia, North Africa and Central Europe (Figure 4). Nonetheless, the analyses showed that China may be a hotspot for the diversification of DHAV-1, with at least 60 years of evolution of the major genetic groups (GG) and subgroups (SG) of DHAV1 [34]. Analyzing sequences of the Hungarian DHAV-1 strains, the only representative sequences from Europe, suggested that DHAV-1 was introduced on at least four occasions during the 2004-2005 Hungarian outbreak; their common ancestors had an estimated TMRCA in the late 1990s and shared an origin with multiple minor Chinese clusters whose representative strains circulated in the early 2000s and 2010s. All these strains belonged to SG 1.3; the viral genomic sequences within SG 1.3 shared 95.8-99.9\% identities. The first group of Hungarian sequences (MT856996 and MT856998) showed a maximum of 99.4\% identity with the closest-related reference sequences, whereas sequence identities were 99.2\% (MT856991, MT856994, MT856995, and MT856997) and 99\% (MT856992, MT856993, MT856999 and MT857000) for other groups of Hungarian isolates. Additionally, sequences collected from heterologous hosts clustered in a common branch (within GG 3) in the time-calibrated phylogenetic tree, a cluster which is estimated to have separated from other GG 3 strains in the late 1970s. This cluster mainly contains sequences collected after 2010 and, referring to the host spectrum, might more readily jump from the common host to a heterologous host. Another genetic group, GG 4, evolved independently from GG 1 to GG 3 strains, and contains now multiple strains from Egypt and China. 


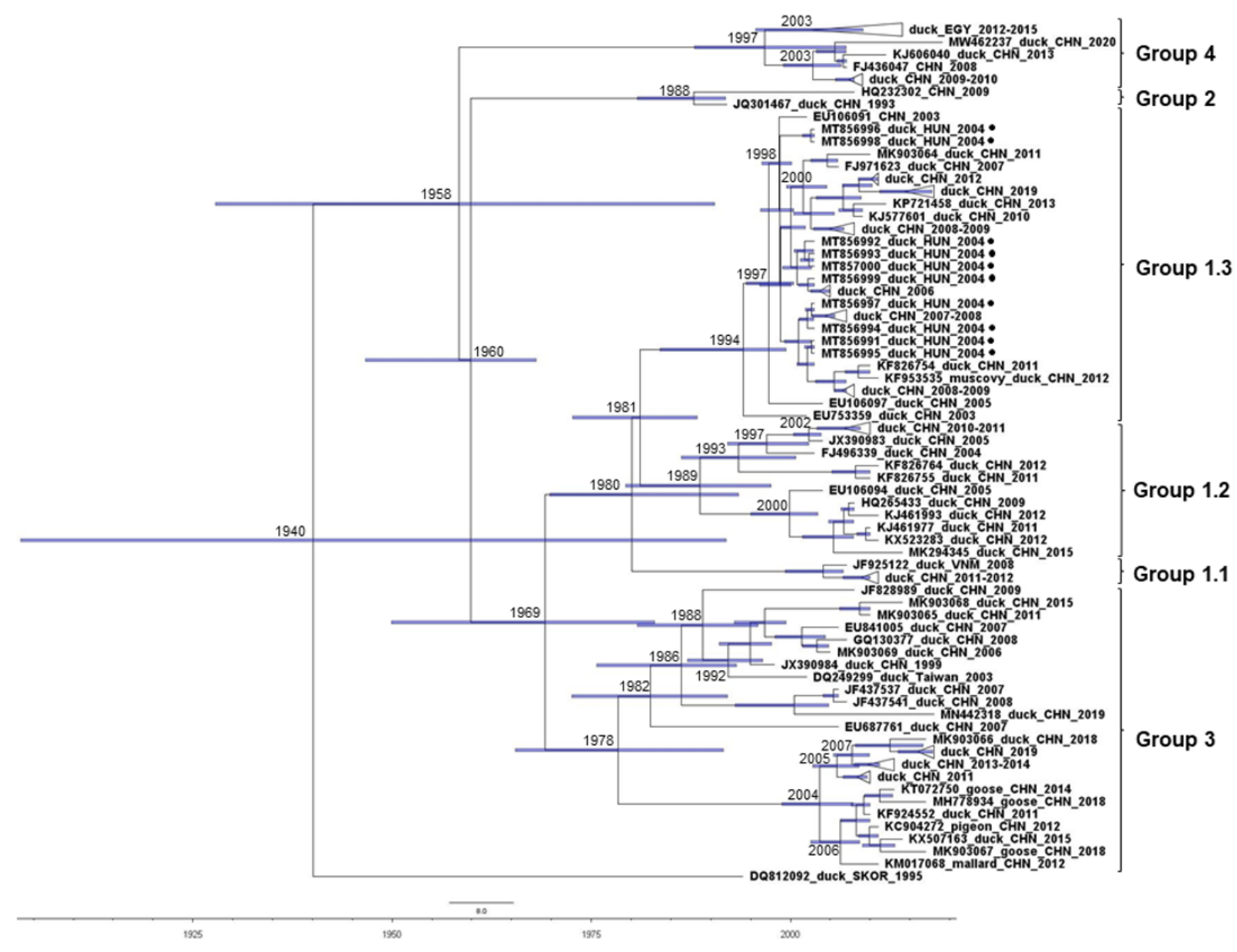

Figure 4. Time-calibrated maximum clade credibility tree of 102 VP1 sequences generated by the BEAST package 1.10.4, $\mathrm{HKY}+\mathrm{G}$ model. Genetic groups and subgroups are on the right.

\section{Conclusions}

Evolutionary mechanisms of DHAV are similar to other picornaviruses; the main features shared by these viruses include high evolutionary rates and the potential for viral RNA recombination, even among heterotypic strains $[32,33,35]$. The evolutionary history and the supporting epidemiological data suggest that Asia may act as a diversification hotspot for DHAVs. The European DHAV-1 strains (represented by the genomic sequences of Hungarian isolates) are closely related to DHAV-1 strains collected in China. This is consistent with earlier observations on other closely related waterfowl origin pathogens whose geographical distribution is shared between these distant geographic areas; all these findings may suggest common drivers or modes of dispersal of a variety of waterfowl viruses between Far East Asia and parts of Europe [36-38]. However, currently available data are scant, which prevents detailed analyses on the route of strain dispersal between Asia and Europe; therefore, further data are greatly needed. Long-distance migrating wild birds along the circumpolar migratory route could mediate the spread of some viruses, including DHAV, known to infect waterfowls [36], although it is unknown whether any of the potential hosts susceptible to DHAV use this migratory route. The trade of domestic ducks and some ornamental bird species may also be a risk for geographical dispersal. Among heterologous hosts susceptible to DHAV, one needs to mention some columbiform and additional anseriform birds (including geese and Muscovy ducks) $[1,18,39,40]$. On the other hand, the routine vaccination of exported domestic breeding ducks and ducklings against DHAV-1 probably results in a reduced risk for virus spread. Concerning other DHAV serotypes, DHAV-2 and, until more recently, DHAV-3, were known to occur only in Asia. The emergence of DHAV-3 in Egypt is, however, an alarming perception. In this 
context, it must be kept in mind that DHAV is prone to intergenotypic recombination, and the timely detection of such recombinants is paramount for disease monitoring. In future surveillance of DHAV infections, genomic epidemiology will serve as a useful tool to track the emergence of recombinant strains with altered phenotypic features and the spread of novel non-indigenous DHAV genotypes.

Author Contributions: Conceptualization, K.B. (Krisztián Bányai); methodology, K.B. (Krisztián Bányai), S.J., K.B. (Krisztina Bali) and Á.B.; software, E.F., S.J., E.K., B.N. and K.I.; formal analysis, E.F., S.J., E.K., B.N. and K.I.; investigation, E.F., S.J., B.N. and K..; resources, K.B. (Krisztián Bányai), Á.B. and V.P.; data curation, Á.B., S.J., E.K. and B.N.; writing-original draft preparation, E.F. and K.B.; writing-review and editing, K.B. (Krisztián Bányai), Á.B., V.P.; supervision, K.B. (Krisztián Bányai); project administration, K.B. (Krisztián Bányai); funding acquisition, K.B. (Krisztián Bányai). All authors have read and agreed to the published version of the manuscript.

Funding: This research was funded by the Hungarian Scientific Research Fund, K120201.

Institutional Review Board Statement: Not applicable.

Informed Consent Statement: Not applicable.

Data Availability Statement: The GenBank accession number of sequences characterized in this study are MT856991-MT857000.

Conflicts of Interest: The authors declare no conflict of interest. The funders had no role in the design of the study; in the collection, analyses, or interpretation of data; in the writing of the manuscript, or in the decision to publish the results.

\section{References}

1. Hisham, I.; Ellakany, H.F.; Selim, A.A.; Abdalla, M.A.M.; El-Abideen, M.A.Z.; Kilany, W.H.; Ali, A.; Elbestawy, A.R. Comparative pathogenicity of duck hepatitis A virus-1 isolates in experimentally infected Pekin and Muscovy ducklings. Front. Vet. Sci. 2020, 7, 234. [CrossRef]

2. Levine, P.P.; Fabricant, J. A hitherto-undescribed virus disease of ducks in North America. Cornell Vet. 1950, 40, 71-86.

3. Liu, R.; Shi, S.; Huang, Y.; Chen, Z.; Chen, C.; Cheng, L.; Fu, G.; Chen, H.; Wan, C.; Fu, Q. Comparative pathogenicity of different subtypes of duck hepatitis A virus in Pekin ducklings. Vet. Microbiol. 2019, 228, 181-187. [CrossRef] [PubMed]

4. Niu, Y.; Ma, H.; Ding, Y.; Li, Z.; Sun, Y.; Li, M.; Shi, Y. The pathogenicity of duck hepatitis A virus types 1 and 3 on ducklings. Poult. Sci. 2019, 98, 6333-6339. [CrossRef] [PubMed]

5. Niu, Y.; Liu, B.; Sun, C.; Zhao, L.; Chen, H. Construction of the recombinant duck enteritis virus delivering capsid protein VP0 of the duck hepatitis A virus. Vet. Microbiol. 2020, 249, 108837. [CrossRef]

6. Kim, M.C.; Kwon, Y.K.; Joh, S.J.; Lindberg, A.M.; Kwon, J.H.; Kim, J.H.; Kim, S.J. Molecular analysis of duck hepatitis virus type 1 reveals a novel lineage close to the genus Parechovirus in the family Picornaviridae. J. Gen. Virol. 2006, 87, 3307-3316. [CrossRef]

7. Tseng, C.H.; Knowles, N.J.; Tsai, H.J. Molecular analysis of duck hepatitis virus type 1 indicates that it should be assigned to a new genus. Virus Res. 2007, 123, 190-203. [CrossRef]

8. Hassan, T.I.R.; Eid, A.A.M.; Ghanem, I.A.I.; Shahin, A.M.; Adael, S.A.A.; Mohamed, F.F. First report of duck hepatitis A virus 3 from duckling flocks of Egypt. Avian Dis. 2020, 64, 269-276. [CrossRef]

9. Kim, M.C.; Kwon, Y.K.; Joh, S.J.; Kim, S.J.; Tolf, C.; Kim, J.H.; Sung, H.W.; Lindberg, A.M.; Kwon, J.H. Recent Korean isolates of duck hepatitis virus reveal the presence of a new geno- and serotype when compared to duck hepatitis virus type 1 type strains. Arch. Virol. 2007, 152, 2059-2072. [CrossRef]

10. Tseng, C.H.; Tsai, H.J. Molecular characterization of a new serotype of duck hepatitis virus. Virus Res. 2007, 126, 19-31. [CrossRef]

11. The Picornavirus Page. Available online: https://www.picornaviridae.com/sg4/avihepatovirus/avihepatovirus_a/dhav-1_seq. htm (accessed on 14 May 2021).

12. Doan, H.T.T.; Le, X.T.K.; Do, R.T.; Hoang, C.T.M.; Nguyen, K.T.; Le, T.H. Molecular genotyping of duck hepatitis A viruses (DHAV) in Vietnam. J. Infect. Dev. Ctries. 2016, 10, 988-995. [CrossRef]

13. Kamomae, M.; Kameyama, M.; Ishii, J.; Nabe, M.; Ogura, Y.; Iseki, H.; Yamamoto, Y.; Mase, M. An outbreak of duck hepatitis A virus type 1 infection in Japan. J. Vet. Med. Sci. 2017, 79, 917-920. [CrossRef]

14. Kozdruń, W.; Czekaj, H.; Lorek, M. Outbreak of duck viral hepatitis in duckling flocks in Poland. Bull. Vet. Inst. Pulawy 2014, 58, 513-515. [CrossRef]

15. Kaleta, E.F. Duck viral hepatitis type 1 vaccination: Monitoring of the immune response with a microneutralisation test in Pekin duck embryo kidney cell cultures. Avian Pathol. 1988, 17, 325-332. [CrossRef]

16. MacPherson, L.W.; Avery, R.J. Duck virus hepatitis in Canada. Can. J. Comp. Med. Vet. Sci. 1957, 21, 26-31.

17. Nikita, N.V.; Leonov, I.K.; Yavdoshak, L.I. Interferonogenic activity of the strain BH-3 duck hepatitis virus type I (Picornaviridae: Avihepatovirus: Avihepatovirus A). Vopr. Virusol. 2021, 66, 162-166. (In Russian) [CrossRef] 
18. Palya, V.; Ivanics, E.; Glavits, R.; Skare, J.; Nagy, E.; Bakonyi, T.; Bajusz, I. Reoccurrence of duck viral hepatitis epidemicspossibilities for prevention and control. Magy Állatorvosok Lapja 2006, 128, 281-287. (In Hungarian)

19. Larsson, A. AliView: A fast and lightweight alignment viewer and editor for large data sets. Bioinformatics 2014, 30, 3276-3278. [CrossRef]

20. Tamura, K.; Stecher, G.; Peterson, D.; Filipski, A.; Kumar, S. MEGA6: Molecular Evolutionary Genetics Analysis version 6.0. Mol. Biol. Evol. 2013, 30, 2725-2729. [CrossRef]

21. Martin, D.P.; Murrell, B.; Golden, M.; Khoosal, A.; Muhire, B. RDP4: Detection and analysis of recombination patterns in virus genomes. Virus Evol. 2015, 1, vev003. [CrossRef]

22. Weaver, S.; Shank, S.D.; Spielman, S.J.; Li, M.; Muse, S.V.; Kosakovsky Pond, S.L. Datamonkey 2.0: A modern web application for characterizing selective and other evolutionary processes. Mol. Biol. Evol. 2018, 35, 773-777. [CrossRef]

23. Rambaut, A.; Lam, T.T.; Carvalho, L.M.; Pybus, O.G. Exploring the temporal structure of heterochronous sequences using TempEst. Virus Evol. 2016, 2, vew007. [CrossRef]

24. Suchard, M.A.; Lemey, P.; Baele, G.; Ayres, D.L.; Drummond, A.J.; Rambaut, A. Bayesian phylogenetic and phylodynamic data integration using BEAST 1.10. Virus Evol. 2018, 4, vey016. [CrossRef]

25. Trifinopoulos, J.; Nguyen, L.T.; von Haeseler, A.; Minh, B.Q. W-IQ-TREE: A fast online phylogenetic tool for maximum likelihood analysis. Nucl. Acids Res. 2016, 44, W232-W235. [CrossRef]

26. Lefort, V.; Longueville, J.E.; Gascuel, O. SMS: Smart Model Selection in PhyML. Mol. Biol. Evol. 2017, 34, 2422-2424. [CrossRef]

27. Rambaut, A.; Drummond, A.J.; Xie, D.; Baele, G.; Suchard, M.A. Posterior summarisation in Bayesian phylogenetics using Tracer 1.7. Syst. Biol. 2018, 67, 901-904. [CrossRef]

28. Molecular Evolution, Phylogenetics and Epidemiology. Available online: http://tree.bio.ed.ac.uk/software/figtree/ (accessed on 14 May 2021).

29. Liu, G.; Wang, F.; Ni, Z.; Yun, T.; Yu, B.; Huang, J.; Chen, J. Genetic diversity of the VP1 gene of duck hepatitis virus type I (DHV-I) isolates from Southeast China is related to isolate attenuation. Virus Res. 2008, 137, 137-141. [CrossRef]

30. Ma, X.; Sheng, Z.; Huang, B.; Qi, L.; Li, Y.; Yu, K.; Liu, C.; Qin, Z.; Wang, D.; Song, M.; et al. Molecular Evolution and Genetic Analysis of the Major Capsid Protein VP1 of Duck Hepatitis A Viruses: Implications for Antigenic Stability. PLoS ONE 2015, 10, e0132982. [CrossRef]

31. Zhang, R.; Yang, Y.; Lan, J.; Xie, Z.; Zhang, X.; Jiang, S. Evidence of possible vertical transmission of duck hepatitis A virus type 1 in ducks. Transbound. Emerg. Dis. 2020, 68, 267-275. [CrossRef]

32. Kuroda, M.; Niwa, S.; Sekizuka, T.; Tsukagoshi, H.; Yokoyama, M.; Ryo, A.; Sato, H.; Kiyota, N.; Noda, M.; Kozawa, K.; et al. Molecular evolution of the VP1, VP2 and VP3 genes in human rhinovirus species C. Sci. Rep. 2015, 5, 8185. [CrossRef]

33. Lu, L.; Dung, N.V.; Bryant, J.E.; Carrique-Mas, J.; Cuong, N.V.; Anh, P.H.; Rabaa, M.A.; Baker, S.; Simmonds, P.; Woolhouse, M.E. Evolution and phylogeographic dissemination of endemic porcine picornaviruses in Vietnam. Virus Evol. 2016, 2, vew001. [CrossRef] [PubMed]

34. Wen, X.; Zhu, D.; Cheng, A.; Wang, M.; Chen, S.; Jia, R.; Liu, M.; Sun, K.; Zhao, X.; Yang, Q.; et al. Molecular epidemiology of duck hepatitis a virus types 1 and 3 in China, 2010-2015. Transbound. Emerg. Dis. 2018, 65, 10-15. [CrossRef] [PubMed]

35. Lukashev, A.N. Recombination among picornaviruses. Rev. Med. Virol. 2010, 20, 327-337. [CrossRef] [PubMed]

36. The Global Consortium for H5N8 and Related Influenza Viruses. Role for migratory wild birds in the global spread of avian influenza H5N8. Science 2016, 354, 213-217. [CrossRef]

37. Kaszab, E.; Lengyel, G.; Marton, S.; Dán, Á.; Bányai, K.; Fehér, E. Occurrence and genetic diversity of CRESS DNA viruses in wild birds: A Hungarian study. Sci. Rep. 2020, 10, 7036. [CrossRef]

38. Kaszab, E.; Marton, S.; Dán, Á.; Farsang, A.; Bálint, Á.; Bányai, K.; Fehér, E. Molecular epidemiology and phylodynamics of goose haemorrhagic polyomavirus. Transbound. Emerg. Dis. 2020, 67, 2602-2608. [CrossRef]

39. Liu, M.; Li, X.; Zhang, Z.; Liu, S.; Zhang, Y. Goose haemorrhagic hepatitis caused by a new subtype duck hepatitis type 1 virus. Vet. Microbiol. 2011, 152, 280-283. [CrossRef]

40. Shi, S.; Chen, H.; Chen, Z.; Fu, G.; Wan, C.; Huang, Y.; Lin, S.; Cheng, L.; Fu, Q.; Lin, J.; et al. Complete genome sequence of a duck hepatitis a virus 1 isolated from a pigeon in China. Genome Announc. 2013, 1, e00451-13. [CrossRef] 